\title{
Inhalt, Vol. 5, No. 6, 1982
}

\section{Contents}

Hinweise für Autoren 266

Sonderbände 266

Impressum 267

Franke, F. und Lampert, F., Gießen

Chromosomenuntersuchungen beim metastasierten Neu-

roblastom 268

Sagaster, P.; Flamm, J. und Micksche, M.,

Wien

Chemoimmunotherapie disseminierter maligner Hoden-

tumoren 273

Bühríng, M. und Menzel, L , Frankfurt/M.

Gewebeschäden nach paravenöser Zytostatikaapplika-

tion - Eine zusätzliche Behandlung mit transkutaner

elektrischer Nervenstimulation - 280

Hausdorf, G.; Erttmann, R.; Hübner, C. und

Winkler, K., Hamburg-Eppendorf

Sonographische Befunde beim malignen lymphoblasti-

schen T-Zell-Lymphom des Kindesalters 284

Vollmoeller, W., Düsseldorf

Ambulante Einzelpsychotherapie bei Krebspatienten . . 293

Onkologie-Mitteilungen 299

Buchbesprechungen 311

Instructions for Authors

266

Special Editions 266

Imprint267

Franke, F. and Lampert, F., Gießen

Chromosomal Analysis in MetastaticNeuroblastoma. . . 268

Sagaster, P.; Flamm, J. and Micksche, M.,

Wien

Chemoimmunotherapy in Disseminated Malignant Testi-

cular Tumors 273

Bühring, M. and Menzel, /., Frankfurt!M.

Soft Tissue Damage after Cytostatic Drug Extravasation

- An Additional Treatment with Transcutaneous Electri

cal Nerve Stimulation -

280

Hausdorf, G.; Erttmann, R.; Hübner, C. and

Winkler, K., Hamburg-Eppendorf 
Sonographic Findings in Children with Lymphoblastic

T-Cell Lymphoma 284

Vollmoeller, W., Düsseldorf

Individual Psychotherapy for Out-Patients with Cancer. . 293

Oncology Communications 299

Book Reviews 311

Beilagen-Hinweise:

Diese Ausgabe enthält eine Beilage der PINO Pharmazeutische Präparate GmbH. Wìr bitten um gfl. Beachtung.

Bibliographischer Hinweis: Inhaltsverzeichnisse dieser Zeitschrift erscheinen regelmäßig in current contents ${ }^{\circledR}$ sowie in anderen bibliographischen Diensten. 